\title{
Departments of clinical pharmacology and therapeutics as sources of data
}

\author{
A. Her Xheimer \\ M.B., B.S., F.R.C.P. \\ Charing Cross Hospital Medical School, London W6 8RF
}

\begin{abstract}
Summary
The functions of a clinical pharmacologist are described and some questions worth asking when prescribing drugs are listed and briefly discussed.
\end{abstract}

A CLINICAL pharmacologist is both a pharmacologist and a clinician. His most important function is to be available for consultation on any medical problem concerned with drugs, in the same way as a gastroenterologist is available for consultation on gastroenterological problems. There are, however, two differences between the clinical pharmacologist and the gastro-enterologist ; firstly, drug-related problems are liable to affect the great majority of patients in hospital and, for that matter, outside hospital, whereas gastro-enterological problems affect a much smaller proportion of patients; secondly, most general practitioners and non-specialist physicians know what gastro-enterological problems they can cope with and at what point they need advice from a specialist, whereas the majority of prescribers are much less aware of the limitations of their knowledge of therapeutics, or of pharmacological problems that may arise in their practice.

A clinical pharmacologist's advice may be sought about the treatment of individual patients. For example, he may be asked what is the best choice of drug for a particular purpose, how the dosage should be handled, and what is the most practical way of assessing the effectiveness of treatment in a patient. Or he may be asked whether it is worth measuring plasma concentrations of the drug and, if so, how the results should be interpreted. If he has the facilities he may make the measurements himself, but his advice is also of value if the chemical pathologist makes them.

The clinical pharmacologist is the most appropriate person to be consulted about policies of drug use in general. For example, the hospital medical committee may ask him to work out rational policies for the use of hypnotics, diuretics, antibiotics, etc., just as a chemical pathologist might be asked to work out guidelines for the use of various liver function tests in different clinical situations.

Another area that concerns the clinical pharmacologist is the detection, reporting and investigation of adverse drug reactions. Far too little attention is paid to these in most hospitals and general practices, $\vec{\omega}$ as a result much information is lost that could be $\frac{\sigma}{0}$ important for individual patients and for the practice of medicine. Clinicians need frequently to be $\frac{3}{-5}$. reminded and encouraged to note and report adverse $\mathrm{c}$ drug effects and to modify their treatment appro- $N$ priately. In some instances it may be best to alter the $\mathcal{G}$ dose or frequency of medication, in others to sub- $O$ stitute a different drug, and so on.

The planning and execution of clinicial trials is another of the clinical pharmacologist's activities. $\vec{c}$ Although he will not necessarily be directly involved $\mathbb{D}$ in all the clinical trials carried out in his hospital, $\mathbb{D}$ his experience makes him a useful person to consuif $\bar{\sigma}$ right from the start, not only from the strictly pharmacological point of view but often also $\overrightarrow{0}$ ethical and statistical aspects.

The clinical pharmacologist also has an important teaching role. This is obvious in a teaching hospital with undergraduate medical students but it is equally important in relation to the junior medical staff, and to a less extent in relation to the pharmacists and nurses.

The emphasis of this symposium is on information $\overrightarrow{\vec{O}}$ and data, but the author believes that clinical 3 pharmacologists should not be regarded primarily as sources of data. Their prime function is to give? advice, that is to make judgments based on applying 을 their specialist experience to the problems that arise in treatment. Frequently they will not know 'the $\frac{5}{3}$ answer' to a question, but they will know where to look for one, and they will be able to suggest possible $\stackrel{\rho}{\rho}$ ways of elucidating and perhaps solving the problems presented to them. This approach is also $\frac{\text { o }}{2}$ embodied in their teaching role: much of the time $\rightarrow$ they can teach students and junior doctors how to solve for themselves the therapeutic problems they $\mathrm{G}$ meet. One way of facilitating this is to provide a list of questions (e.g. see below) which students and 0 doctors can usefully ask themselves about drug treatment (Herxheimer, 1976; 1977).

(1) Need. Is the drug really needed? What is likely to happen if it is not used?

(2) Class. To what class does the drug belong? $\stackrel{?}{+}$ (e.g. a potassium-sparing diuretic; a phenothiazine) 
(3) Aim. What aim is to be achieved with the drug? What disorder of function is to be corrected, or what symptom relieved? When are the effects of treatment expected to begin?

(4) Observations. What observations should be made to judge whether the aim has been achieved? How should serious unwanted effects be watched for? When should these observations be made, and by whom?

(5) Route and dosage. By what route, in what dose, at what intervals and at what times is the drug to be given, and why? Up to what limit is it worth increasing dosage and/or frequency if the response is inadequate?

(6) Alternatives. What other drugs could be used instead of this one? Do the drugs differ notably in efficacy or safety? Do their costs differ much?

(7) Duration. How long should the drug continue to be used, and how will the decision be made to stop?

(8) Unwanted effects. What undesirable effects may occur from the drug? Are they acceptable? What is their approximate frequency? How can they be avoided, or treated if they occur?

(9) Elimination. How is the drug eliminated? Will the patient's illness change the usual pattern of distribution and effects of the drug? If yes, how does this affect the dosage?

(10) Interactions. Are there any other drugs, foods or activities which the patient should avoid while he is receiving the drug?
(11) Patient's ideas. What does the patient believe about the drug? What has he been told about it and what does he remember? Does he need additional information? Will he take the drug?

Some of the questions can be answered by a pharmacist, since they concern the pharmaceutical and pharmacological properties of the drug. These are the questions about the class to which the drug belongs (2), the route and dosage (5), unwanted effects (8), drug elimination (9) and interactions (10). But the questions about the need for treatment (1), its aim (3) and the observations to be made (4) and the duration of treatment (7) are better answered by the clinician concerned, if necessary by discussion with a clinical pharmacologist. The questions which the clinical pharmacologist will need to help with most often are those about alternative remedies (6) and probably questions 4 and 9 . These are questions where facts are necessary but are not in themselves enough, because an evaluation of the facts is required. The clinical pharmacologist thus in no way competes with the information pharmacist: they complement one another, and ideally every district hospital should have one of each.

\section{References}

HerXheimer, A. (1976) Towards parity for therapeutics in clinical teaching. Lancet, ii, 1186.

HerXheimer, A. (1977) Some questions worth asking about what we prescribe. Drug and Therapeutics Bulletin, 15, 17. 